\title{
Three axis vector magnet set-up for cryogenic scanning probe microscopy
}

\author{
J. A. Galvis,,$^{1,2}$ E. Herrera, ${ }^{1}$ I. Guillamón, ${ }^{1,3}$ J. Azpeitia, ${ }^{3,4}$ R. F. Luccas, ${ }^{3,4}$ C. Munuera, ${ }^{3,4}$ \\ M. Cuenca, ${ }^{5}$ J. A. Higuera, ${ }^{5}$ N. Díaz, ${ }^{5}$ M. Pazos, ${ }^{5}$ M. García-Hernandez,${ }^{3,4}$ A. Buendía, ${ }^{1}$ \\ S. Vieira, ${ }^{1,3}$ and H. Suderow ${ }^{1,3}$ \\ ${ }^{1}$ Laboratorio de Bajas Temperaturas, Departamento de Física de la Materia Condensada, Instituto de Ciencia \\ de Materiales Nicolás Cabrera, Condensed Matter Physics Center (IFIMAC), Facultad de Ciencias \\ Universidad Autónoma de Madrid, 28049 Madrid, Spain \\ ${ }^{2}$ Departamento de Ciencias Naturales Facultad de Ingeniería Universidad Central, Bogotá, Colombia \\ ${ }^{3}$ Unidad Asociada de Bajas Temperaturas y Altos Campos Magnéticos, UAM, CSIC, Cantoblanco, \\ E-28049 Madrid, Spain \\ ${ }^{4}$ Instituto de Ciencia de Materiales de Madrid, Consejo Superior de Investigaciones Científicas (ICMM-CSIC), \\ Sor Juana Inés de la Cruz 3, 28049 Madrid, Spain \\ ${ }^{5}$ SEGAINVEX, Universidad Autónoma de Madrid, 28049 Madrid, Spain
}

(Received 19 November 2014; accepted 22 December 2014; published online 14 January 2015)

\begin{abstract}
We describe a three axis vector magnet system for cryogenic scanning probe microscopy measurements. We discuss the magnet support system and the power supply, consisting of a compact three way 100 A current source. We obtain tilted magnetic fields in all directions with maximum value of $5 \mathrm{~T}$ along $\mathrm{z}$-axis and of $1.2 \mathrm{~T}$ for XY-plane magnetic fields. We describe a scanning tunneling microscopy-spectroscopy (STM-STS) set-up, operating in a dilution refrigerator, which includes a new high voltage ultralow noise piezodrive electronics and discuss the noise level due to vibrations. STM images and STS maps show atomic resolution and the tilted vortex lattice at $150 \mathrm{mK}$ in the superconductor $\beta-\mathrm{Bi}_{2} \mathrm{Pd}$. We observe a strongly elongated hexagonal lattice, which corresponds to the projection of the tilted hexagonal vortex lattice on the surface. We also discuss Magnetic Force Microscopy images in a variable temperature insert. (o 2015 AIP Publishing LLC. [http://dx.doi.org/10.1063/1.4905531]
\end{abstract}

\section{INTRODUCTION}

In a number of systems of topical interest, such as anisotropic superconductors or magnets, it is important to control the vector of the magnetic field. Macroscopic measurements, such as resistivity, susceptibility, thermal conductivity, or specific heat, are made in tilted magnetic fields by using rotating sample holders. ${ }^{1,2}$ They provide information about transport or thermodynamic properties as a function of the tilt of the magnetic field. For example, in unconventional superconductors with gap nodes, specific heat or thermal conductivity experiments in tilted magnetic fields are used to tilt vortices with respect to the crystalline lattice, with the aim to obtain the location of the gap nodes. ${ }^{2}$ Exploring the vortex lattice properties under tilted magnetic fields can be used to establish internal anisotropies and observe possible vortex lattice transitions in the mixed state of superconductors. ${ }^{3-5}$ In these, and other cases, it would be useful to obtain local information by making imaging experiments using scanning probe microscopy. 6,7

Scanning probe microscopes are, however, very sensitive to mechanical vibrations and require a mechanically stable platform. ${ }^{8-13}$ Thus, to control the direction of the magnetic field, rotating the microscope is not a good solution, and it is better to use superconducting three axis coil systems. Hess et al. used a three axis magnet and a dilution refrigerator to study the tilted vortex lattice in superconductors in a pioneering experiment. ${ }^{5}$ But since then, few scanning probe experiments in vectorial magnetic field systems have been reported. Here, we describe a three axis coil system that has been successfully used in conjunction with a dilution refrigerator scanning tunneling microscopy (STM)/S and a liquid helium temperatures Magnetic Force Microscopy (MFM). We show atomic resolution images and spectroscopic maps of superconducting vortex lattice in inclined fields at $150 \mathrm{mK}$ obtained with the STM. We describe details of vibration isolation and electronics (superconducting coil power supply and high voltage piezodrives) of the dilution refrigerator STM. We also present MFM measurements in a magnetic recording disc.

\section{THE MAGNET AND CRYOGENIC SETUP}

The space needed for a scanning probe microscope head often exceeds $40 \mathrm{~mm}$ in diameter, requiring three axis superconducting coil systems with clear bore above $50 \mathrm{~mm}$. The field homogeneity must be good, so that magnetic field gradients exert no force on sensitive parts of the scanning probe microscope. The support system of the magnet is also important, because the magnet assembly is heavy, and should not move to avoid vibrations on the microscope head.

We have built a magnet consisting of five coils, one solenoid for $\mathrm{z}$ axis and two sets of split coils for the XY plane (Fig. 1(a)). The inside bore is fixed by the z-axis coil, of $56 \mathrm{~mm}$, and the outside diameter of the magnet is $250 \mathrm{~mm}$. The five 
a

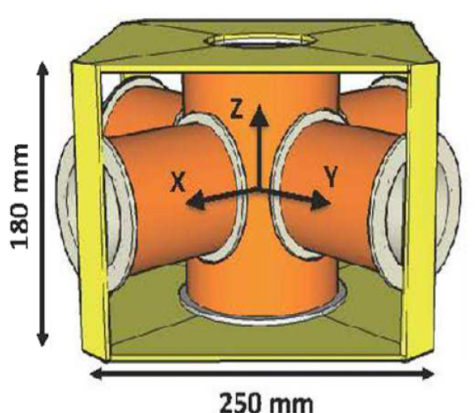

b

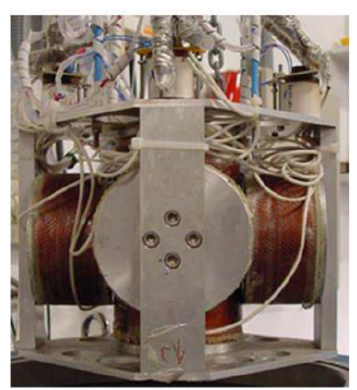

C

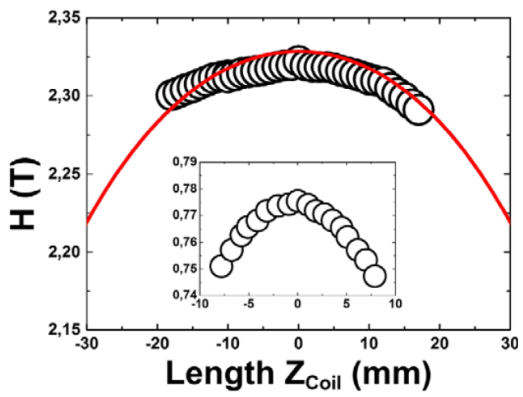

FIG. 1. (a) Schematic view of the three axis magnet. One long coil is used to generate the z-axis magnetic field. For the in-plane field, we use two crossed split coil systems centered on the z-axis coil. The support is made of Al. Each coil is connected to a superconducting switch. In (b), we show a photograph of the magnet, viewed from the side. Superconducting switches are located on top of the magnet. Superconducting cabling is made continuous as far as possible. In particular, wiring is continuous for each of the split coils. The magnet hangs from the top of the cryostat, as shown in Fig. 2(b). In (c), we show the magnetic field vs z-axis position, with respect to the center of the magnet when the z-coil is energized (50 A) (main panel) and when the $\mathrm{x}$ or $\mathrm{y}$ coils are energized (75 A, inset). Red line is a guide to the eye.

coils are mounted in an $\mathrm{Al}$ cage designed to hold the forces which are generated by the interaction between coils. We chose Al to reduce as far as possible the weight of the magnet. We used stainless steel M6 screws to fix the magnets and $1 \mathrm{~cm}$ thick Al plates for the magnet cage (Fig. 1(b)). The free space in between coils is filled using Poly (Vinyl Chloride) pieces in order to eliminate as far as possible unused liquid helium volume around the magnet (not shown in Fig. 1(b)).

We reach a maximum magnetic field of $5 \mathrm{~T}$ in the $\mathrm{z}$ axis and $1.2 \mathrm{~T}$ in the $\mathrm{x}-\mathrm{y}$ plane, using a current of about $100 \mathrm{~A}$. This gives a tilted magnetic field of $5 \mathrm{~T}$ at a cone with opening angle of $13.5^{\circ}$ around $\mathrm{z}$. The cone increases its opening angle towards lower fields on the $\mathrm{z}$-axis coil, and we reach the full three dimensional vector capability for fields below $1.2 \mathrm{~T}$. We have measured the magnetic field as a function of the distance and find a homogeneous field within a sphere of $0.5 \mathrm{~cm}$ radius around the center of the coil system of $0.2 \%$ for the magnetic field along the $\mathrm{z}$ axis, and of $1 \%$ for the magnetic field in the plane (Fig. 1(c)). The three coil systems are equipped each with a persistent mode switch, which allows maintaining the magnetic field constant when the liquid helium is above the magnet.

The magnet is immersed in liquid helium and hangs from a room temperature flange (Fig. 2(b)) using four stainless steel bars $4 \mathrm{~mm}$ in diameter. The magnet is energized using three pairs of copper strands. Further wiring includes voltage drop measurements at the superconducting switch on each coil, and connections to the persistent mode switch heaters. The copper strands consist of bunches of several tens copper wires each $0.5 \mathrm{~mm}$ in diameter, which are fixed at each radiation shield. The total diameter of copper wire is halved at the radiation shield located at approximately the nitrogen temperature shield of the dewar and again when reaching the highest admissible helium level. Below this point, we use, in parallel to the copper strands, a NbTi superconducting cable. When we modify the magnetic field, we heat all three persistent mode switch heaters, with a liquid helium consumption of $1.5 \mathrm{l} / \mathrm{h}$. For STM experiments, we usually work in the persistent mode, with the switch heaters off. So, during normal operation, the liquid helium consumption is below $0.9 \mathrm{l} / \mathrm{h}$ and the hold time of the cryostat is 4 days. For the room temperature connectors for the current leads, we use four way Jaeger ${ }^{\mathrm{TM}}$ leak tight high current connectors.

With the aim to have a compact measurement system with the lowest possible noise levels, we have also designed and built a new magnet power supply (Fig. 3(a)). We use three independent current sources. Each one has a commuted internal commercial stage of $5 \mathrm{~V} 100 \mathrm{~A}$, followed by a voltage to current converter consisting of a stage providing linear regulation which uses MOSFET power transistors. The linear stage employs a Hall sensor to measure the current flowing through the load circuit. We compare the measured current with the set-point given by the user. To adjust the current, we use a PI control that has been optimized to work with a high inductive load. The PI controls the gate of the linear MOSFET stage. The linear stage provides excellent noise levels. It requires, however, a high power dissipation of $500 \mathrm{~W}$ per current supply. In total, we have to dissipate about $1500 \mathrm{~W}$. To avoid cumbersome wind channels and fans, we use water cooling with conventional microprocessor closed circuit coolers. This requires heat exchangers at the linear stage and then another heat exchanger with a small fan. We use a power dissipation system available in the market as microprocessor coolers. We verified that the Hall sensor is insensitive to the stray field of the coils when the power supply is located at $2 \mathrm{~m}$ from the superconducting coil. Overcurrent protection is made with Schottky diodes. We use a 16 bit digital to analog converter and obtain a resolution in current of about $3 \mathrm{~mA}$.

\section{DILUTION REFRIGERATOR STM SET-UP}

The STM head is similar to the one described in Ref. 14. We use a helium3-helium4 dilutor refrigerator Kelvinox MX100 15 with $100 \mu \mathrm{W}$ cooling power at $100 \mathrm{mK}$. The STM head and dilution refrigerator is housed inside a vacuum chamber made of $\mathrm{Al}$ immersed in the liquid helium bath of the magnet. The STM is located on a copper support system anchored at the mixing chamber Figs. 2(a) and 2(c).

It turned out to be important to tightly fix the magnet. Otherwise, we observed that the pendular motion of the magnet was transmitted to the STM. We surrounded the 
a.

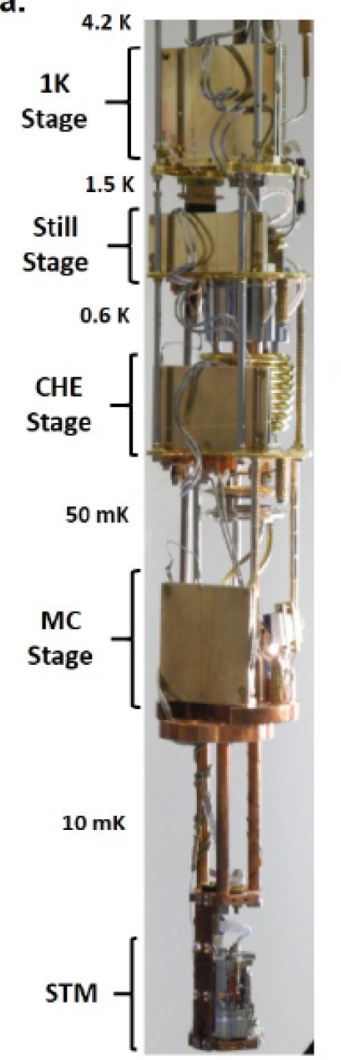

b.

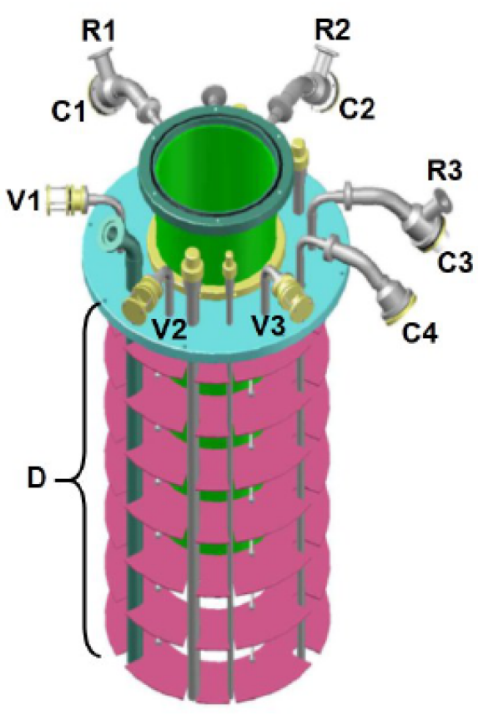

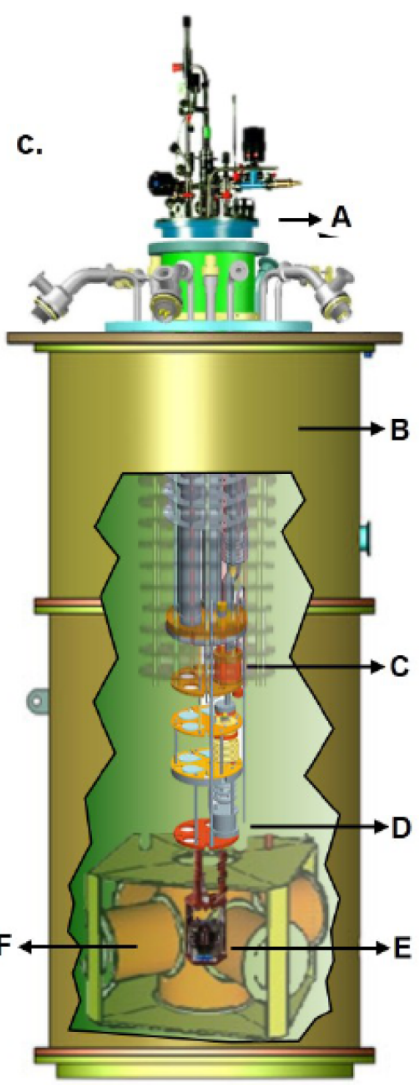

FIG. 2. In (a), we show a photograph of the dilution refrigerator insert. We mark all cooling stages. At each stage, we use large golded plates to thermalize the cables. The microscope is located at the center of the magnet. It is posed on a copper support, using fiberglass to decouple it and reduce the vibration level at the STM head. In (b), we show the magnet support system with the upper cable connectors (C1-C3 for current supply and C4 for diagnosis of the coil and temperature measurement), helium recovery points (R1-R3), and security valves (V1-V3). We use several stainless steel screens (D). All tubes have small lateral holes to avoid Taconis vibrations. In (c), we show the whole cryostat. The dilution refrigerator (A) hangs from the top, the dewar (B), the $1 \mathrm{~K}$ pot $(\mathrm{C})$, the mixing chamber (D), the STM head (E), and the superconducting coils (F).

magnet with fiberglass hoses to contact the magnet with the inside wall and the bottom of the helium dewar. The inner vacuum chamber of the insert is also tightly fitted into the magnet, and the whole dewar is hanged from the roof using flexible ropes.

One relevant source of noise in the STM measurements is the high voltage lines that drive the piezos. To minimize noise in these lines, we have designed a new high voltage amplifier (Fig. 3(b)). To choose the best high voltage operational amplifier, we used a conventional noise test-bed, providing the noise level of an op-amp between $0.1 \mathrm{~Hz}$ and $10 \mathrm{~Hz}$. We measured a single line high voltage amplifier PA90 and the more cost effective double line PA243 amplifier. We obtained noise levels 50 times higher for the PA243, which is in addition ten times more sensitive to temperature drifts than the PA90. To reduce the noise level of the PA243, we tried to fix the input of the PA243 by adding a stage with a low noise level PA27. With this, we managed to reduce the noise level to half the value found in the PA90. However, the input stage requires frequency compensation, and it turned out to be quite difficult to avoid overshoots and unwanted high frequency effects. Thus, we finally chose the single line high voltage amplifier PA90 and worked on the design of the rest of the electronics to achieve best possible performance.

We designed a compact system, including power supply circuits, for multiple signal entry and four $\pm 150 \mathrm{~V}$ high voltage lines in a single box. We took special care to avoid line crossing and to provide good grounding planes well distributed over the circuitry (Fig. 3(b)). But this is not enough to extract the lowest possible noise level. Temperature stability of the electronics becomes important. Even minute air flow can produce changes in the DC voltage of same order than the noise. Temperature dependence of offset voltage can be easily of several tens of $\mu \mathrm{V} /{ }^{\circ} \mathrm{C}$. Thermoelectric voltages also influence low frequency noise levels, as well as temperature variations in the power supply.

Thus, we fixed all op amps to a single Al block. We made a temperature compensation unit, which maintains the temperature of the op amps at $45^{\circ} \mathrm{C}$ using a linear regulator and a slow and low noise ventilator for cooling. The ventilator does not interfere into any amplifier channel. We took special care of the stability of the power supply, which is of $0.05 \% /{ }^{\circ} \mathrm{C}$. We reached a noise level of $3.75 \mu \mathrm{V}$ peak to peak between $0.1 \mathrm{~Hz}$ and $10 \mathrm{~Hz}$. The bandwidth of the high voltage signals goes up to $100 \mathrm{kHz}$. Noise levels provided by similar high voltage amplifiers available in the market are mostly above this value.

In Fig. 4, we show measurements of the noise level at several stages of the STM set-up. We measure the vertical displacement with an accelerometer in different positions, and then the displacement of the tip with respect to the sample in the STM head. In the room where all pumps needed to 
a)
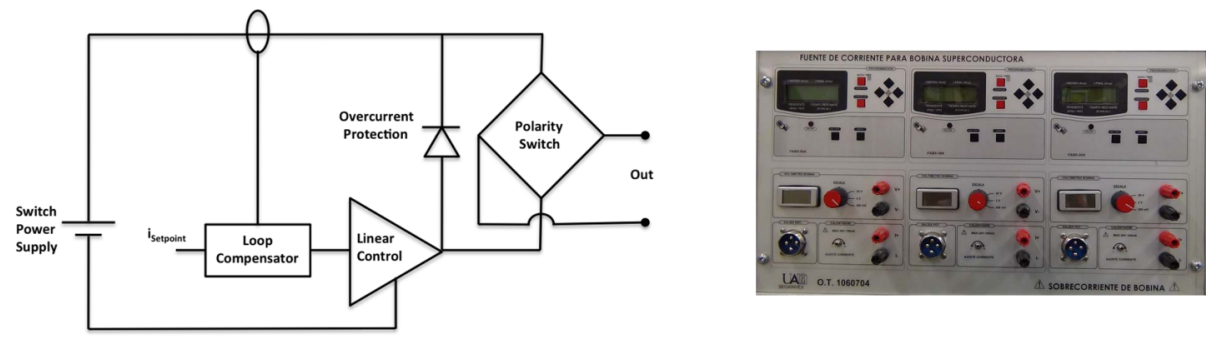

b)

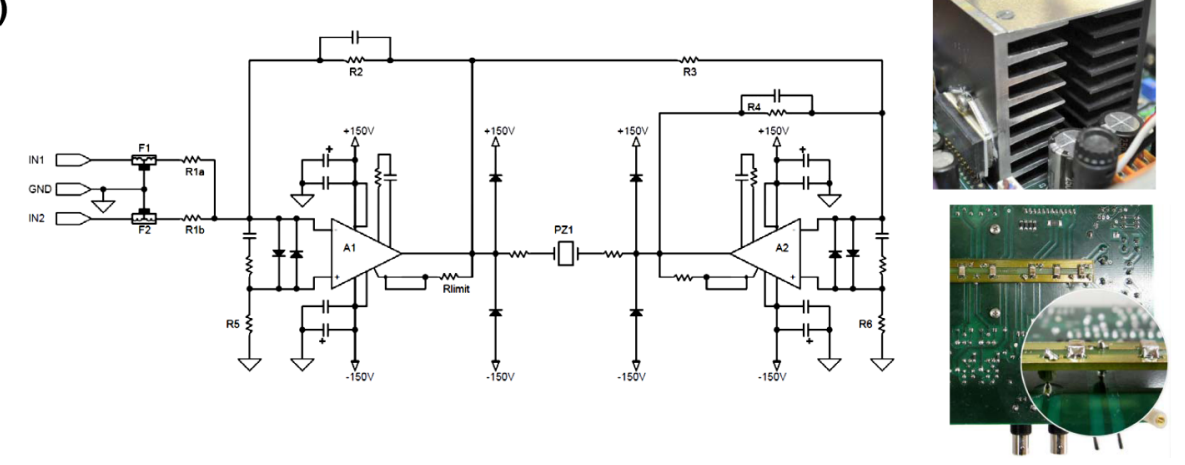

FIG. 3. In (a), we show a scheme of the current power supply for the magnet, as discussed in the text (left panel). In the right panel, we show a photograph of the power supply. It is rather compact, $50 \mathrm{~cm}$ high and $80 \mathrm{~cm}$ long. In (b), we show in the left panel, a basic scheme for high voltage amplifiers (A1 and A2) in opposition to drive a piezo. This is the most simple arrangement, and includes filters at the entrance and basic overvoltage protection. We take utmost care of the ripple of the power supply, and of the temperature control. We cool the amplifiers using a large refrigerator shown in the upper right panel. We arrange all elements of the electronics in such a way as to avoid crosstalk and noise. In the lower right panel, we show a motive of the high voltage power supply bus.

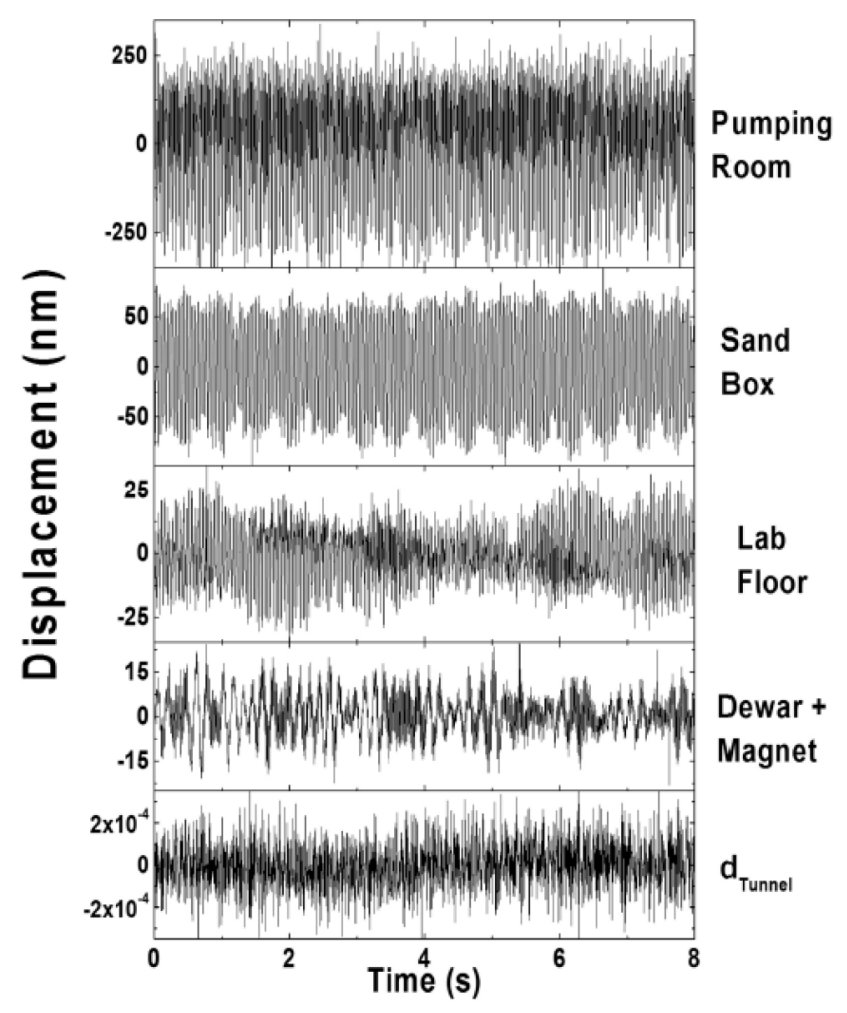

FIG. 4. Displacements induced by vibrations at different parts of the dilution refrigerator experimental set-up. The upper four curves are taken using an accelerometer. The lower curve is obtained by measuring the STM current as a function of time without the STM feedback loop. operate the dilution refrigerator are located, we find vibration amplitudes of $300 \mathrm{~nm}$ as measured on top of the pumps.

The pumping pipes from the pumping room to the fridge are wide diameter stainless steel tubes connected in series with at least $2 \mathrm{~m}$ long PVC hoses reinforced with a steel spiral, which go through one sand box. At the sand box, we find vibration levels of about $50 \mathrm{~nm}$. Between the sandbox and the cryostat, we continue with $1 \mathrm{~m}$ long PVC hoses. The helium3-helium4 mixture is pumped through the PVC hoses, without any remarkable effect on the operation of the dilution refrigerator. After a few days, we clean the nitrogen traps to reduce contamination due to degasing. We use the hoses armorvin $^{\mathrm{TM}}$ described in Ref. 16. Within the dewar on top of the magnet, we find a vibration level of about $15 \mathrm{~nm}$, which is not modified when we switch the pumps off.

The vibration level on the laboratory floor is somewhat larger, of about $25 \mathrm{~nm}$, and is due to residual motion of the base of the building and to acoustical noise. To reduce the part corresponding to acoustical noise, we glue Copopren ${ }^{\mathrm{TM}}$ to the outside of the dewar. The dewar is screened from the vibrations of the building by simply using flexible ropes on which we hang the dewar. To reduce further the sensitivity of the STM head to vibrations, we pose the microscope on fiberglass on top of its support anchored at the mixing chamber.

We measure the vibration of the tip over the sample using the tunneling current with the standard expression $I_{\text {tunnel }} \propto e^{-2 \kappa d}$ with $\kappa=\sqrt{\frac{2 m \phi}{\hbar^{2}}}$ ( $m$ being the free electron mass, $\phi \approx 5 \mathrm{eV}$ the work function of $\mathrm{Au}$, and $d$ the distance between tip and sample). We use tip and sample of gold and find 
a)

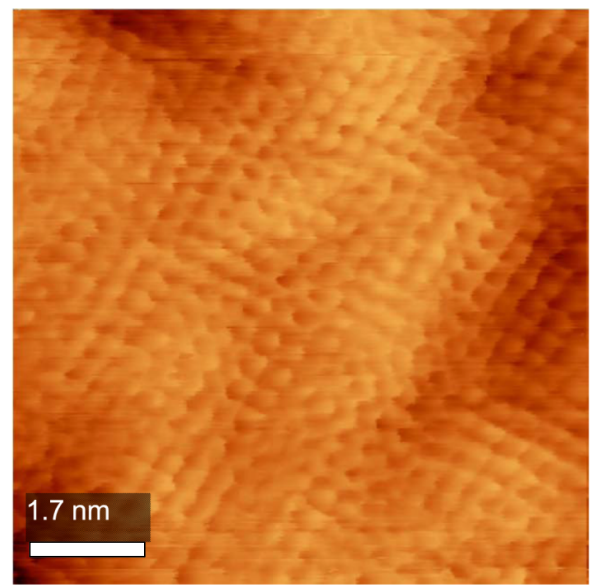

b)
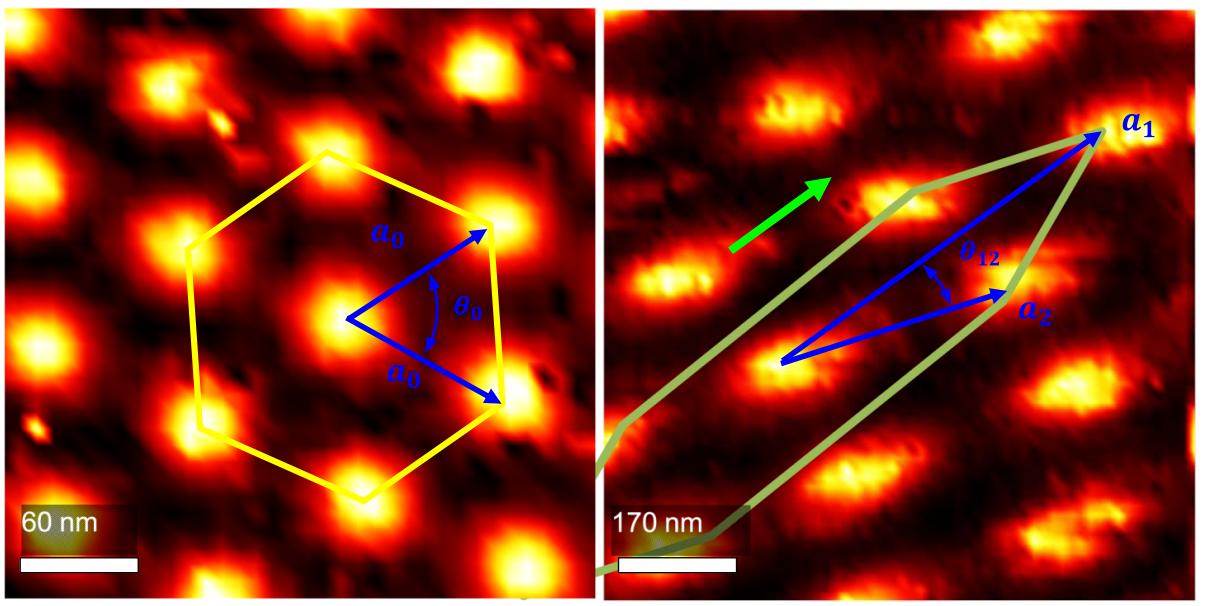

c)

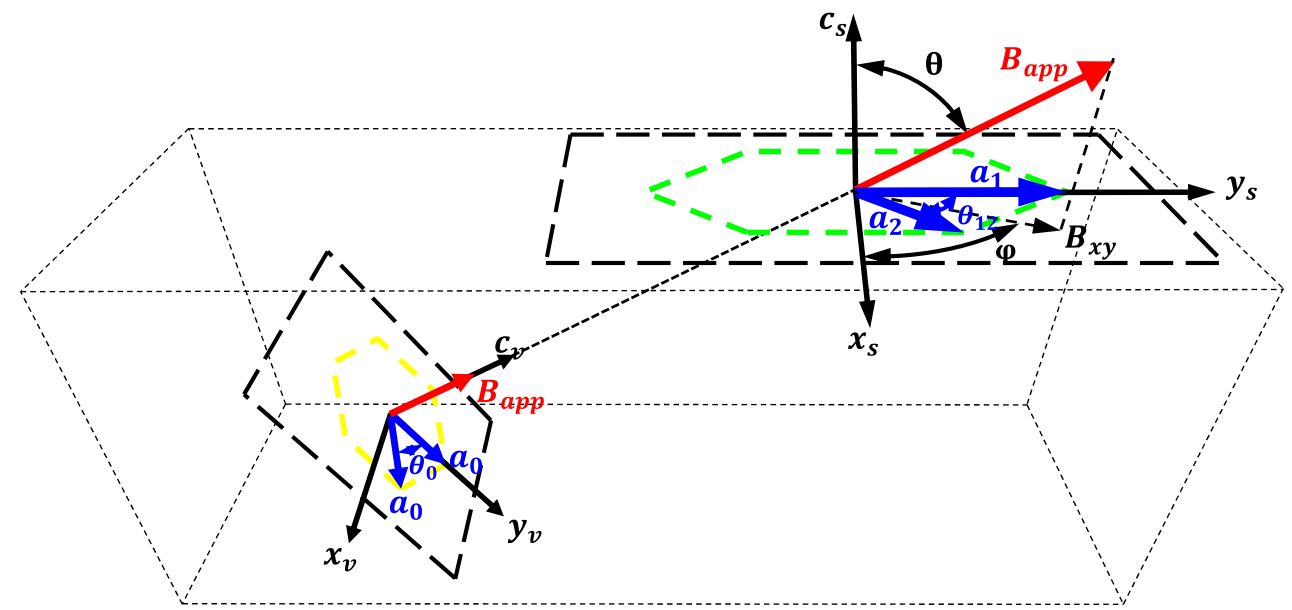

FIG. 5. In (a), we show atomic patterns of the square Bi lattice in $\beta$ - $\mathrm{Bi}_{2} \mathrm{Pd}$ at $150 \mathrm{mK}$. In (b), we show the vortex lattice with perpendicular field, along the c-axis of the crystal structure (at $0.3 \mathrm{~T}$, left panel, yellow hexagon show the vortex lattice unit cell). Right panel shows the vortex at the surface when the magnetic field is tilted (polar angle $\theta=80^{\circ}$ and azimuthal angle $\phi=34^{\circ}$ ). The green arrow gives the magnetic field projection in the plane and the green hexagon, the unit cell of the surface projection of the vortex lattice. In (c), we show schematically the vortex lattice frame (yellow) and the surface frame (green) as well as relevant angles and unit cell vectors.

values below $0.2 \mathrm{pm}$ at $100 \mathrm{mK}$ with the dilution refrigerator operating and the magnet energized.

The voltage noise level of our electronics mentioned above produces displacements of the piezotube one order of magnitude below this value (using a piezotube with displacement $100 \AA / \mathrm{V}$ in the $\mathrm{x}-\mathrm{y}$ plane). Thus, noise in the tunneling current is due to residual vibrations.

\section{RESULTS ON THE SUPERCONDUCTING VORTEX LATTICE IN TILTED MAGNETIC FIELDS USING DILUTION REFRIGERATOR STM/S}

In order to check the operation of the system, we performed STM/S measurements on the superconductor $\beta$ $\mathrm{Bi}_{2} \mathrm{Pd}$. We obtained atomic resolution and observed the tilted superconducting vortex lattice. In Fig. 5, we show atomic 
a)
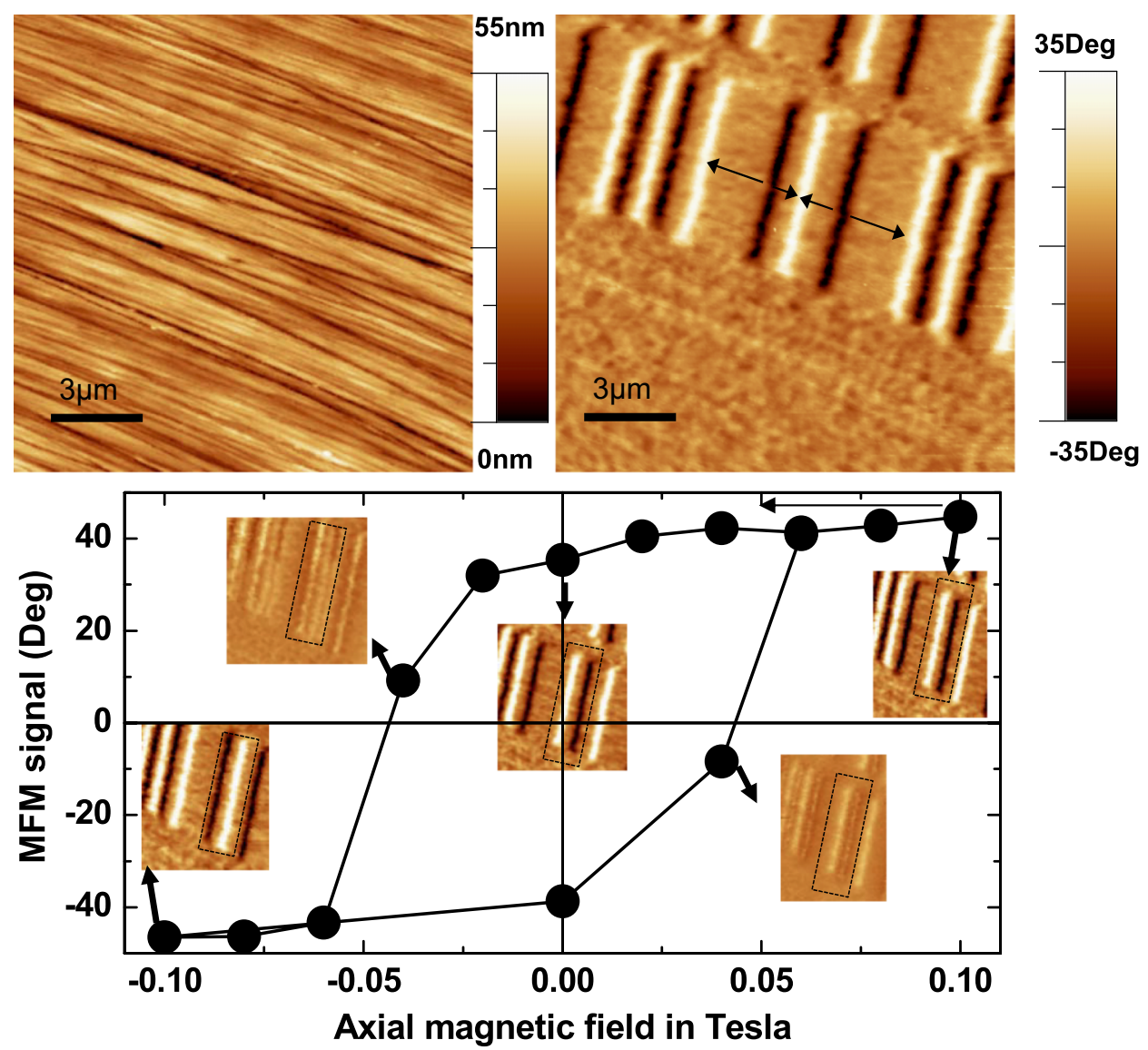

b)
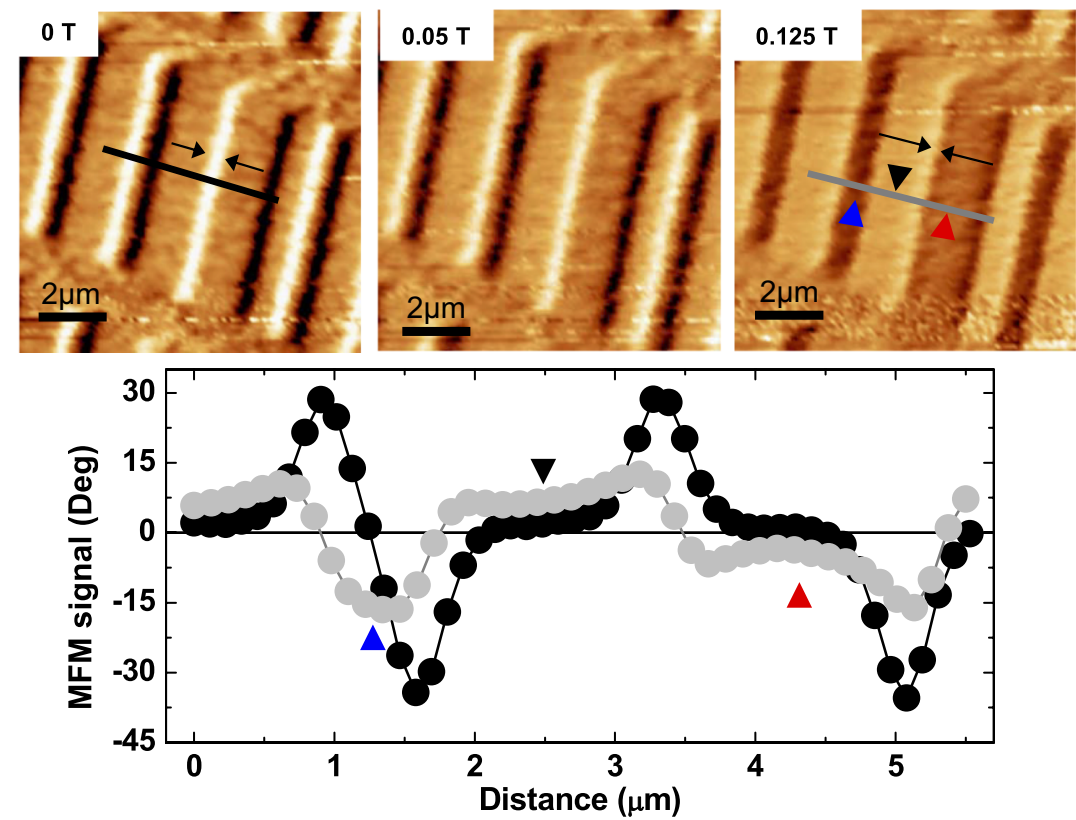

FIG. 6. (a) Simultaneous topographic (left) and magnetic (right) images of a magnetic recording disk. Black arrows indicate the magnetization direction within the sample. Bottom panel shows axial hysteresis loops of the MFM probe deduced from images acquired at different applied fields at the same position. Insets show the images. (b) MFM images for different in-plane fields, always at the same location. Black arrows indicate the magnetization direction within the sample. Bottom panel shows line profiles (marked in the MFM images) showing the differences in contrast, measured when the probe magnetization direction switches from axial to transversal.

resolution images and conductance maps at zero bias of superconducting vortex lattice in tilted fields in $\beta$ - $\mathrm{Bi}_{2} \mathrm{Pd}$ obtained at $150 \mathrm{mK}$. To make the conductance maps, we use the same methods as in previous work. ${ }^{17}$ The features of the atomic lattice (Fig. 5(a)) and the measurement of the superconducting gap and of the hexagonal Abrikosov lattice when the field is normal to the surface (Fig. 5(b) left panel) will be discussed in detail elsewhere. ${ }^{18}$ The intervortex distance 
$a_{0}$ agrees with Abrikosov lattice $a_{0}=\left(\frac{4}{3}\right)^{\frac{1}{4}} \sqrt{\frac{\Phi_{0}}{B}}$ (where $\Phi_{0}$ is the flux quantum and $\mathrm{B}$ the applied magnetic field).

In the right panel of Fig. 5(b), we show the vortex lattice in tilted magnetic fields. The vortex lattice stretches in the direction of the field inclination. Ginzburg-Landau and London equations superconductors show that the vortex lattice at the surface is the geometrical projection of the vortex lattice in the bulk. ${ }^{3}$ Previous measurements of the tilted vortex lattice were made on the strongly anisotropic superconductor $2 \mathrm{H}-\mathrm{NbSe}_{2}$. Its vortex lattice is strongly distorted in tilted magnetic fields due to the layered structure of the crystal lattice. ${ }^{5}$ In plane and out of plane critical fields $H_{c 2, a b}$ and $H_{c 2, c}$ are very different in that compound $\left(H_{c 2, a b} \approx 3 * H_{c 2, c}\right)$. When the magnetic field is not along the $\mathrm{c}$ axis, the vortex lattice is no longer a simple hexagon. The distortion can be described by an ellipse which encircles the hexagon. The long axis of the ellipse is of $a_{0} / \gamma$, with $\gamma=\left(1+\left(\frac{H_{c 2, a b}}{H_{c 2, c}}\right)^{2} \tan ^{2}(\theta)\right)^{1 / 4} \cos ^{1 / 2}(\theta)$ (with $\theta$ the polar angle). $\beta-\mathrm{Bi}_{2} \mathrm{Pd}$ is only slightly anisotropic, with $H_{c 2, a b}=1.2 * H_{c 2, c} \cdot{ }^{18}$ We find thus $\gamma=0.92$ when $\theta=80^{\circ}$. The elliptical vortex lattice observed at the surface is thus the projection of a nearly perfectly hexagonal vortex lattice inside the sample.

It is important to distinguish between the vortex lattice frame and the surface frame (Fig. 5(c)). The projection can produce many different vortex patterns on the surface frame and can be obtained from geometrical calculation. To obtain the result shown in Fig. 5, we first chose an azimuthal angle $\left(\phi=34^{\circ}\right)$ for which the tilt of the magnetic field goes exactly along one of the main axes of the vortex lattice (green arrow in Fig. 5(b)), and then tilt the magnetic field with a polar angle of $\theta=80^{\circ}$. At this azimuthal angle, the long axis of the hexagon at the surface is given by $a=\frac{a_{0} \gamma}{\cos (\theta)}$. For the image in Fig. 5(b), we find an intervortex distance $a_{0}=88 \mathrm{~nm}$ with perpendicular fields (left panel). With the inclined field (right panel), we find $a_{1}=564 \mathrm{~nm}$ for the long axis of the vortex lattice in the surface frame, which gives $\theta=81.7^{\circ}$. Thus, there is a difference of about $2^{\circ}$ between the angle provided by the three axis coil system and the angle obtained from the shape of the vortex lattice. This difference can be due to a slight misalignment of the sample's surface with respect to the three axis coil.

\section{MFM IN THE VECTOR MAGNET}

We finally present results in a commercial MFM system from Nanomagnetics Instruments operated inside a variable temperature insert immersed in the liquid helium bath of the magnet. MFM measurements of a magnetic recording disk are shown in Fig. 6. We use magnetic probes of Nanosensors (PPP-MFMR). To measure simultaneously topography and magnetic profile, we first make a line scan to obtain topographic information using the scanning force microscopy. Then, we retract the tip $50 \mathrm{~nm}$, and rescan the profile previously measured in magnetic force mode. The magnetic contrast is provided by the gradient of the force between tip and sample at $50 \mathrm{~nm}$ height. ${ }^{19}$ In Fig. 6(a), we show the topography and the magnetic image of a hard disk with parallel magnetic domains and axial tip magnetization. MFM image reveals a linear pattern of black and white stripes perpendicular to the recording track of the disk. There is no correlation with the topographic image.

MFM contrast in recording media is well understood and results from the interaction of the local surface field component along the direction of the probes magnetization. ${ }^{20-22}$ When the MFM probe is axially oriented, the contrast (black and white lines) provides the transition regions between bits. ${ }^{23}$

When we modify the applied magnetic field perpendicular to the surface and switch its direction, we reorient the magnetization of the tip, and obtain its axial hysteresis loop (Fig. 6(a)). Starting with a saturated state, $+0.1 \mathrm{~T}$, we decrease the magnetic field in steps of $0.02 \mathrm{~T}$, down to $-0.1 \mathrm{~T}$, and then back to positive values. Typically, the tip magnetization switches at $\pm 0.04 \mathrm{~T}^{24}$

With an in-plane magnetic field, we can switch between axial and transversal probe magnetization (Fig. 6(b)). Note that, due to the shape anisotropy of the tip, this is only possible with in-plane magnetic fields. We find that increasing the magnetic field along one direction in plane, changes the MFM contrast from bright and dark strips, located at the transition regions between bits, to fully bright and dark regions within each bit, depending on the writing state. ${ }^{21}$ For the axial magnetic switching field, we find $0.1 \mathrm{~T}$.

\section{SUMMARY AND CONCLUSIONS}

Three axis magnetic field measurements in local probe systems are a research area likely to grow in the near future. Cryogenic scanning probe systems are readily available in many different forms, and there are many materials requiring a controlled vector magnetic field. Here, we have described a STM set-up, for which vibrations are most critical. We also discuss a simple solution to make a compact power supply, with excellent noise performance and a newly designed high voltage amplifier providing noise levels below $10 \mu \mathrm{V}$. The noise at the STM head is due to vibrations, which we have characterized and reduced using simple means, as PVC hoses for helium 3 pumping and sand boxes. We present results of STM in superconductors and MFM in magnetic systems. The anisotropic features with respect to the tilt of the magnetic field are observed in the vortex lattice and in magnetic patterns.

\section{ACKNOWLEDGMENTS}

This work was supported by Convocatoria Doctorados en el Exterior 568-2012 COLCIENCIAS, the Spanish MINECO (FIS2011-23488, MAT2011-27470-C02-02, CSD2009-00013), by the Comunidad de Madrid through program Nanofrontmag-CM (S2013/MIT-2850) and by Marie-Curie actions under the project FP7-PEOPLE-2013CIG-618321. The research leading to these results has received funding from the European Union Seventh Framework Programme under Grant Agreement No. 604391 Graphene Flagship. We also acknowledge Banco Santander, COST MP1201. J.A. and C.M. acknowledge the FPI (BES2012-058600) and Juan de la Cierva (JCI-2011-08815) programs, respectively. 
${ }^{1}$ H. Suderow, J. Brison, A. Huxley, and J. Flouquet, J. Low Temp. Phys. 108, 11 (1997)

${ }^{2}$ K. Izawa, H. Takahashi, H. Yamaguchi, Y. Matsuda, M. Suuki, T. Sasaki, T. Fukase, Y. Yoshida, R. Settai, and Y. Onuki, Phys. Rev. Lett. 86, 2653 (2001).

${ }^{3}$ L. J. Campbell, M. M. Doria, and V. G. Kogan, Phys. Rev. B 38, 2439 (1988).

${ }^{4}$ V. G. Kogan, Phys. Rev. B. 51, 15344 (1995).

${ }^{5}$ H. F. Hess, C. Murray, and J. V. Waszczak, Phys. Rev. B 50, 16528 (1994).

${ }^{6}$ J. A. Galvis, H. Suderow, S. Vieira, S. L. Budko, and P. C. Canfield, Phys. Rev. B 87, 214504 (2013).

${ }^{7}$ J. Galvis, L. Chirolli, I. Guillamón, S. Vieira, E. Navarro-Moratalla, E. Coronado, H. Suderow, and F. Guinea, Phys. Rev. B 89, 224512 (2014).

${ }^{8}$ U. R. Singh, M. Enayat, S. C. White, and P. Wahl, Rev. Sci. Instrum. 84, 013708 (2013).

${ }^{9}$ M. Assig, M. Etzkorn, A. Enders, W. Stiepany, C. R. Ast, and K. Kern, Rev. Sci. Instrum. 84, 033903 (2013).

${ }^{10}$ S. Misra, B. B. Zhou, I. K. Drozdov, J. Seo, L. Urban, A. Gyenis, S. C. J. Kingsley, H. Jones, and A. Yazdani, Rev. Sci. Instrum. 84, 103903 (2013).

${ }^{11}$ Y. J. Song, A. F. Otte, V. Shvarts, Z. Zhao, Y. Kuk, S. R. Blankenship, A. Band, F. M. Hess, and J. A. Stroscio, Rev. Sci. Instrum. 81, 121101 (2010).
${ }^{12}$ S. H. Pan, E. W. Hudson, and J. C. Davis, Rev. Sci. Instrum. 70, 1459 (1999).

${ }^{13}$ H. Suderow, I. Guillamón, J. G. Rodrigo, and S. Vieira, Supercond. Sci. Technol. 27, 063001 (2014).

${ }^{14}$ H. Suderow, I. Guillamón, and S. Vieira, Rev. Sci. Instrum. 82, 033711 (2011).

${ }^{15}$ See http://www.oxford-instruments.com for more information about Oxford Instruments NanoScience, 2014.

${ }^{16}$ See http://www.merlett.com/Products-list/ARMORVIN-HNA for more information about Merlett Tecnoplastic S.p.A., 2014.

${ }^{17}$ I. Guillamón, H. Suderow, F. Guinea, and S. Vieira, Phys. Rev. B 77, 134505 (2008).

${ }^{18} \mathrm{E}$. Herrera et al., "Magnetic field dependence of the density of states in the multiband superconductor $\beta$ - $\mathrm{Bi}_{2} \mathrm{Pd}$ " (unpublished).

${ }^{19}$ X. Zhu and P. Grutter, IEEE Trans. Magn. 39, 3420 (2003).

${ }^{20}$ D. Rugar, H. Mamin, P. Guethner, S. Lambert, J. Stern, C. McFadyen, and T. Yogi, J. Appl. Phys. 68, 1 (1990).

${ }^{21}$ R. Gomez, E. Burke, and I. Mayergoyz, J. Appl. Phys. 79, 6441 (1996).

${ }^{22}$ H. Zhong, W. Peng, G. Tarrach, A. Drechsler, J. Jiang, D. Wei, and J. Yuan, J. Phys. D: Appl. Phys. 41, 085002 (2008).

${ }^{23}$ A. Hubert, W. Rave, and S. Tomlinson, Phys. Status Solidi B 204, 817 (1997).

${ }^{24}$ M. Jaafar, A. Asenjo, and M. Vazquez, IEEE Trans. Nanotechnol. 7, 245 (2008). 